\title{
Leptospirosis ocupacional en una región del Caribe colombiano
}

Saholeth N ájera, Bacteriól, ${ }^{(1)} \mathrm{N}$ elson Alvis, PhD, ${ }^{(2)}$ D avid Babilonia, Bacteriól, ${ }^{(1)}$

Ligia A Ivarez, Bacteriól,(1) Salim Máttar, PhD. (1)

Nájera S,Alvis N, Babilonia D,Alvarez L, Máttar S. Leptospirosis ocupacional en una región del Caribe colombiano. Salud Publica Mex 2005;47:240-244.

\section{R esumen}

Objetivo. Establecer la seroprevalencia ocupacional a la infección por Leptospira en el departamento de Córdoba, Colombia. Material y métodos. Estudio de prevalencia en poblaciones en riesgo del departamento de Córdoba, Colombia, entre febrero y abril de 2004, con el fin de detectar anticuerpos IgM antileptospira en 344 trabajadores agrícolas, carniceros y recolectores de basuras (nivel de confianza $99.9 \%$, error máximo $0.5 \%$, prevalencia $72 \%$ ) y muestreo estratificado proporcional a habitantes y ocupación. U tilizando SPSSS 11.0 para W indows se hizo análisis mediante pruebas no paramédicas (Ji cuadrada) $p<0.05, y$ se calcularon razones de momios con intervalos de confianza. Resultados Prevalencia elevada de antecedentes de infección por Leptospira (13.1\%). N 0 existieron diferencias entre zonas, se observó una asociación por residir en el municipio de Ciénaga de 0 ro a una mayor infección (RM= 3.52 IC $1.70-7.26)(p=0.00283)$. El trabajador agrícola estuvo asociado con mayor infección ( $R M=2.04$ IC $1.080-3,85)$ $(p=0.025)$, así como tomar agua de represa $(R M=2.418$ IC 1.24-4.70) $(p=0.00787)$. Conclusiones La tasa de infección es importante y existe un riesgo considerable para la salud pública en un área específica del Caribe colombiano.

Palabras clave: leptospirosis; sero prevalencia; Colo mbia; Región del Caribe

\author{
Nájera S,Alvis N, Babilonia D, Alvarez L, Máttar S. \\ Occupational leptospirosis \\ in a Colombian Caribbean area. \\ Salud Publica Mex 2005;47:240-244.
}

\section{A bstract}

Objective To establish the seroprevalence of infection by Leptospira in an occupational setting in Cordoba. Material and methods. A cross-sectional study was conducted among 334 farmworkers, butchers, and garbage collectors, to identify the presence of anti-lepto spira IgM antibodies, in the Department of Cordoba, Colombia. Stratified sampling proportional to the number of inhabitants and occupation was used to select the sample population (confidence level $99.9 \%$, error $0.5 \%$, prevalence $72 \%$ ). The SPSS software 11.0 version was used to perform non parametric tests with $p<0.05$, as well as odds ratios with confidence intervals. Results. The prevalence of previous infection by Leptospira was high (13.1\%). No differences among areas were found, however, higher infection was associated with living in Cienaga de 0 ro municipality $(0 R=3.52 \mathrm{Cl} 1.70-7.26)(p=$ 0,00283 ). Being a farmer was also a risk factor for infection $(O R=2.04 \mathrm{Cl} 1.080-3.85)(p=0.025)$, as well as drinking water from a dam $(0 \mathrm{R}=2.4 \mathrm{Cl} 1,24-4,70)(p=0.00787)$. Conclusions The rate of infection is important and a significant public health problem in this area of the Colombian Caribbean coast.

Key words: leptospirosis; seroprevalence; Colombia; Caribean Region

(1) Universidad de Córdoba, Instituto de Investigaciones Biológicas del Trópico, Facultad de Medicina Veterinaria Zootecnia, Montería, Colombia.

(2) U niversidad de Cartagena, Grupo de Investigaciones en Economía de la Salud. Cartagena, Colombia.

Fecha de recibido: 22 de septiembre de 2004 - Fecha de aprobado: 1 de marzo de 2005 Solicitud de sobretiros: Salim Máttar, PhD. Universidad de Córdoba. Instituto de Investigaciones Biológicas del Trópico, Facultad de Medicina Veterinaria y Zootecnia. Montería, Colombia. Correo electrónico: smattar@ escarsa.net.co 
a leptospirosis es una zoonosis remergente de distribución mundial con un comportamiento endémico. La infección representa no sólo un problema con implicaciones epidemiológicas sino económicas y sociales. ${ }^{1,2}$ Es más frecuente en regiones tropicales, sus principales reservorios son animales domésticos que infectan al hombre por exposición directa con tejidos, vísceras, secreciones de animales infectados, contacto con agua o suelos contaminados e incluso a través de heridas o piel intacta. ${ }^{3,4}$ Constituye un serio problema de salud pública, en especial en aquellas zonas con saneamiento básico inadecuado donde las condiciones de trabajo y el tipo de actividad favorecen su desarrollo.

La infección está vinculada principalmente con actividades ocupacionales en los agricultores, trabajadores de alcantarillas y veterinarios susceptibles de adquirirla debido a su alto riesgo de exposición. 5,6 La enfermedad no tiene un cuadro clínico específico pero se relaciona con fiebre, mialgia, cefalea, ictericia o formas anictéricas, hasta formas graves con síndromes renales, hepáticos y hemorrágicos, o enfermedad de Weil. ${ }^{7}$ Los anticuerpos IgM aparecen tres días después de la infección y pueden persistir hasta por cinco meses. ${ }^{8-10}$

En Colombia, entre 2000 y 2003 se conocen sólo dos estudios de prevalencia de leptospirosis; uno en la ciudad de Cali en personas sintomáticas, y el otro en el municipio de Don Matías (Antioquia) en trabajadores agrícolas, con tasas de ataque de 6.4 y $22.8 \% .{ }^{9}, 11$ En el departamento de Córdoba, región ganadera con clima tropical propicio para el desarrollo de esta zoonosis, se cuenta con estudios de prevalencia de leptospirosis en porcinos cuya elevada tasa de infección oscila entre 30 y $50 \% .^{12-14}$

El objetivo del presente estudio fue determinar la seroprevalencia en un grupo de personas con un alto riesgo de exposición a la infección por Leptospira en el departamento de Córdoba, Colombia.

\section{Material y métodos}

Este estudio evaluó la existencia de anticuerpos antileptospira en habitantes de ocho municipios del departamento de Córdoba, entre febrero y abril de 2004. Dicho departamento tiene 28 municipios en $25.020 \mathrm{~km}^{2}$, temperatura de $30{ }^{\circ} \mathrm{C}$, y humedad relativa de $89 \%$. Su territorio forma parte de la llanura Caribe y es atravesado por el río Sinú, que delimita tres zonas (alta, media y baja) de óptimas condiciones para el desarrollo de las actividades agropecuarias.

Se consideró población de estudio a los 961000 habitantes de los municipios del Medio Sinú (Montería, Cereté y Ciénaga de Oro) y Bajo Sinú (Lorica, Cotorra, Purísima, Momil y Chimá). En ésta, se identificaron tres grupos de riesgo laboral (operarios agrícolas o ganaderos, carniceros y recolectores de basura) los cuales integraban una población de 249713 personas. La muestra se calculó mediante el programa EPI Info Versión 6.0, 2000 (CDC, Atlanta Ga, EUA), estimando un nivel de confianza de $99.9 \%$, un error máximo de $0.5 \%$ y una prevalencia esperada de la infección por Leptospira de $12 \%$ en las poblaciones de estudio, de acuerdo con estudios preliminares en la región..$^{15}$ Se hizo un muestreo estratificado proporcional al número de habitantes por municipios y acorde a las actividades realizadas; la selección de los trabajadores al interior de los grupos fue al azar. A todas las personas que participaron se les aplicó una encuesta que incluyó factores de riesgo: ocupación, contacto con animales y la fuente de agua utilizada.

Las muestras de sueros se analizaron en el Instituto de Investigaciones Biológicas del Trópico de la Universidad de Córdoba, mediante un inmuno ensayo para detección de anticuerpos IgM anti-leptospira, en suero, ELISA-IgM. (Panbio limited, Australia ref. E-LEP01M).

Los datos se incorporaron a una base previamente diseñada y los resultados se expresaron en frecuencias absolutas y relativas. Mediante análisis bivariado a través de pruebas no paramétricas se evaluó la asociación entre la presencia de anticuerpos antileptospira y las variables registradas en el formulario (características sociodemográficas generales y antecedentes patológicos y epidemiológicos), se consideró un $p<0.05$ como significativo. Además, se calcularon las razones de momios (RM) e intervalos de confianza respectivos. En el procesamiento de los datos se empleó el paquete estadístico SPSSS 11.0 para Windows.

\section{Resultados}

De los 344 sueros estudiados, $47 \%$ correspondieron a la zona del Bajo Sinú y 53\% al Medio Sinú, la seropositividad fue similar en ambas zonas con prevalencia de $13.1 \%$ (cuadro I). El cuadro II presenta las prevalencias de infección de acuerdo a características generales, relación con animales y antecedentes epidemiológicos. Las diferencias entre zonas no fueron significativas, pero entre municipios se observó asociación entre residencia en el municipio de Ciénaga de Oro y una mayor infección ( $\mathrm{RM}=3.52$ IC 1.70-7.26) ( $p=0.00283)$. La ocupación de operario agrícola o ganadero estuvo asociada a una mayor infección ( $\mathrm{RM}=2.04$ IC 1.080-3.85) $(p=0.025)$. Respecto al abastecimiento de agua, el tomarla de la represa estuvo asociado con mayor infección $(\mathrm{RM}=2.418$ IC 1.24-4.70) ( $p=0.00787)$. En cuanto a la edad y al sexo no hubo diferencias. En relación con ani- 


\section{Seropositividad a Leptospira. Córdoba, Colombia, 2004}

\begin{tabular}{lrr} 
Características sociodemográficas & Frecuencia & $\%$ \\
$\begin{array}{l}\text { Procedencia } \\
\text { Cereté }\end{array}$ & 41 & 11.92 \\
\hline Ciénaga de 0 ro & 48 & 13.95 \\
\hline Montería & 90 & 26.16 \\
\hline Total zona Medio Sinú & 179 & 52.03 \\
\hline Chimá & 10 & 2.91 \\
\hline Cotorra & 30 & 8.72 \\
\hline Lorica & 64 & 18.60 \\
\hline Momil & 36 & 10.47 \\
\hline Purísima & 25 & 7.27 \\
\hline Total zona Bajo Sinú & 165 & 47.97 \\
& & \\
Edad (años) & & \\
15 a 29 & 79 & 22.97 \\
\hline 30 a 44 & 145 & 42.15 \\
\hline 45 y más & 120 & 34.88
\end{tabular}

Sexo

\begin{tabular}{rrr} 
Femenino & 47 & 13.66 \\
\hline Masculino & 297 & 86.34
\end{tabular}

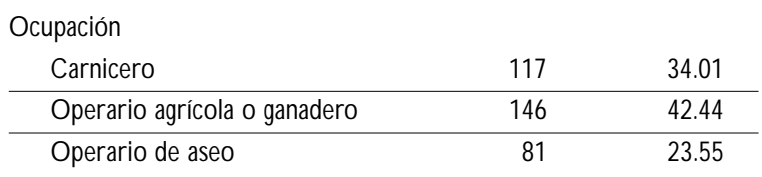

males como perros, ganado bovino, cerdos y roedores no se evidenciaron diferencias significativas respecto a la infección por Leptospira.

\section{Discusión}

En el departamento de Córdoba, así como en Colombia, la leptospirosis es una zoonosis raramente diagnosticada, probablemente por falta de conocimiento de la enfermedad o ausencia de métodos diagnósticos de la infección por Leptospira. El estudio encontró una prevalencia elevada de antecedentes de infección con presencia de anticuerpos IgM en $13.1 \%$ de la población. Esta prevalencia corresponde a la esperada por las condiciones climáticas de la región y las actividades laborales prevalentes. En estudios en Perú, en localidades rurales del departamento de San Martín, cuya actividad ocupacional principal es la agricultura, se encontró una prevalencia de $25.2 \%,{ }^{16}$ mayor que la de nuestro estudio, probablemente debido a precarias condiciones higiénicas y a características geográficas de esa región. Sin embargo, la detección de anticuerpos fue de tipo $\operatorname{IgG}$, lo que sólo indica exposiciones previas a la infección, a diferencia del método utilizado en esta investigación, que detectó la presencia de anticuerpos tipo IgM, lo cual establece infecciones recientes. En la leptospirosis los anticuerpos IgM aparecen tres días después de la infección y pueden persistir hasta por cinco meses, esto presume un alto contacto con el microrganismo de las personas incluidas en el estudio. ${ }^{17,18}$ No obstante, la prevalencia de leptospirosis tanto en Colombia como Perú, Brasil, México y Costa Rica, está alrededor de 14.1 a $16.0 \%,{ }^{16}$ y la enfermedad es de carácter endémico en zonas rurales dedicadas a actividades agropecuarias en condiciones inadecuadas de saneamiento.

La falta de diferencias entre las zonas quizá se explique por las condiciones físicas, ambientales y geográficas similares, lo que indica que la distribución de la enfermedad es homogénea y no representa un factor predisponente para adquirir la infección. Sin embargo, el análisis por municipios indica focalización de la infección y se asocia con la presencia de mayor prevalencia de leptospirosis en animales, como lo muestran estudios previos que destacan una mayor tasa de infección por leptospirosis animal en Ciénaga de Oro y Cotorra. ${ }^{12-14}$ Esto sugiere la relación directa entre la transmisión de la enfermedad y la presentación de la infección en la población estudiada.

La seropositividad entre hombres (13.5\%) y mujeres $(10.6 \%)$, no mostró diferencias, es decir, el sexo no fue un factor determinante para adquirir la infección. La edad tampoco lo fue aunque el estudio encontró que la población seropositiva a Leptospira estuvo principalmente en el rango de edades comprendidas entre 15 a 44 años, lo que se explica por el hecho de ser edades productivas. Los datos anteriores coinciden con los de Meites y colaboradores ${ }^{18}$ en estudios de prevalencia en California entre los años de 1992 a 2001, quienes afirmaron que la leptospirosis tiene una mayor presentación en adultos jóvenes, rango en donde se concentra la mayoría de los individuos dedicados a actividades con gran exposición a la enfermedad. ${ }^{18}$

Respecto de antecedentes patológicos de síntomas ninguno de los estudiados mostró asociación con la infección, probablemente porque la mayor parte de los casos de leptospirosis presentan una sintomatología inespecífica o leptospirosis anictérica o asintomática. ${ }^{19}$ Esto es consistente con lo hallado en otros estudios de seroprevalencia en áreas donde la infección es endémica, con elevadas tasas de infección en individuos con sintomatología inespecífica. ${ }^{20}$ 
Cuadro II

\section{Presencia de anticuerpos antileptospira en relación con características generales, animales y antecedentes epidemiológicos. Córdoba, Colombia, 2004}

Positivo \% Negativo \% p

Características generales

Municipio de residencia

\begin{tabular}{|c|c|c|c|c|c|}
\hline - Cereté & 3 & 7.3 & 38 & 92.7 & \\
\hline - Chimá & 1 & 10.0 & 9 & 90.0 & \\
\hline - Ciénaga de oro & 14 & 29.2 & 34 & 70.8 & 0.00283 \\
\hline - Cotorra & 5 & 16.7 & 25 & 83.3 & \\
\hline - Lorica & 6 & 9.4 & 58 & 90.6 & \\
\hline - Momil & 5 & 13.9 & 31 & 86.1 & \\
\hline - Montería & 7 & 7.8 & 83 & 92.2 & \\
\hline - Purísima & 4 & 16.0 & 21 & 84.0 & \\
\hline \multicolumn{6}{|l|}{ Zona de residencia } \\
\hline • Bajo Sinú & 21 & 12.7 & 144 & 87.3 & NS \\
\hline - Medio Sinú & 24 & 13.4 & 155 & 86.6 & \\
\hline \multicolumn{6}{|l|}{ Edad } \\
\hline - 15 a 29 & 12 & 15.2 & 67 & 84.8 & \\
\hline - 30 a 44 & 19 & 13.1 & 126 & 86.9 & NS \\
\hline - 45 y más & 14 & 11.7 & 106 & 88.3 & \\
\hline \multicolumn{6}{|l|}{ Sexo } \\
\hline - Femenino & 5 & 10.6 & 42 & 89.4 & NS \\
\hline - Masculino & 40 & 13.5 & 257 & 86.5 & \\
\hline \multicolumn{6}{|l|}{ A bastecimiento de agua } \\
\hline - Acueducto & 20 & 9.9 & 183 & 90.1 & \\
\hline - Represa & 17 & 22.1 & 60 & 77.9 & 0.00787 \\
\hline - Pozo & 7 & 12.5 & 49 & 87.5 & \\
\hline - Ciénaga & 1 & 12.5 & 7 & 87.5 & \\
\hline \multicolumn{6}{|l|}{0 cupación } \\
\hline - Carnicero & 8 & 6.8 & 109 & 93.2 & \\
\hline - O perario agrícola o & 026 & 17.8 & 120 & 82.2 & 0.025 \\
\hline - O perario de aseo & 11 & 13.6 & 70 & 86.4 & \\
\hline Total prevalencia & 45 & 13.1 & 299 & 86.9 & \\
\hline
\end{tabular}

N S = no significativo

\author{
Positivo \% Negativo \% p
}

Relación con animales

\begin{tabular}{|c|c|c|c|c|c|}
\hline \multicolumn{6}{|l|}{ Perro } \\
\hline Sí & 19 & 14.5 & 112 & 85.4 & NS \\
\hline No & 26 & 12.2 & 187 & 87.7 & \\
\hline \multicolumn{6}{|l|}{ Cerdo } \\
\hline Sí & 21 & 14.7 & 121 & 85.2 & NS \\
\hline No & 24 & 11.8 & 178 & 88.1 & \\
\hline \multicolumn{6}{|c|}{ Roedores } \\
\hline Sí & 36 & 13.5 & 229 & 86.4 & NS \\
\hline No & 9 & 11.3 & 70 & 88.6 & \\
\hline \multicolumn{6}{|c|}{ Bovinos } \\
\hline Sí & 28 & 12.7 & 192 & 87.2 & NS \\
\hline $\mathrm{No}$ & 17 & 13.7 & 107 & 86.2 & \\
\hline
\end{tabular}

Antecedentes patológicos

Fiebre

\begin{tabular}{crrrrr} 
Sí & 4 & 7.1 & 52 & 92.9 & NS \\
\hline N o & 33 & 12.4 & 233 & 87.6 & \\
Cefalea & & & & &
\end{tabular}

\begin{tabular}{lrrrrr} 
Sí & 9 & 7.9 & 105 & 92.1 & NS \\
\hline N o & 28 & 13.5 & 180 & 86.5 &
\end{tabular}

Dolor muscular

\begin{tabular}{crrrrr} 
Sí & 13 & 12.1 & 94 & 87.9 & N S \\
\hline N o & 18 & 9.5 & 171 & 90.5 & \\
Artralgias & & & & & \\
Sí & 7 & 9.7 & 65 & 90.3 & N S \\
\hline N o & 24 & 10.7 & 200 & 89.3 & \\
Ictericia & & & & &
\end{tabular}

\begin{tabular}{lrrrrr} 
Sí & 1 & 10.0 & 9 & 90.0 & NS \\
\hline $\begin{array}{l}\text { No } \\
\text { Heridas en piel } \\
\text { Sí }\end{array}$ & 30 & 10.5 & 256 & 89.5 & \\
\hline N o & 41 & 14.0 & 251 & 86.0 & NS \\
\hline
\end{tabular}

El grado de exposición ocupacional fue importante, los individuos dedicados a labores agrícolas tuvieron una mayor prevalencia que la obtenida por los trabajadores dedicados al sacrificio de animales y los trabajadores de aseo y alcantarillado. Por tanto, la ocupación de operario agrícola o ganadero asociada a una mayor infección $(\mathrm{RM}=2.04 \mathrm{IC} 1.080-3.85) \quad(p=0.025)$ demuestra el carácter ocupacional de la leptospirosis. En ese sentido, las prevalencias encontradas en trabajadores agrícolas de países como México, Brasil, Cuba y Costa Rica, confirman este hecho. ${ }^{16,17,20}$
Acerca de la fuente de abastecimiento de agua, el tomarla de la represa se asoció con una mayor infección por Leptospira (RM= 2.418 IC 1.24-4.70) ( $p=0.00787)$; esto se explica por el estancamiento del agua de la represa, que favorece la transmisión de la infección y confirma que las condiciones de saneamiento básico, entre ellas la fuente de agua, son un factor determinante de riesgo.

Las investigaciones de seroprevalencia humana en Colombia son escasas; no existen estudios en poblaciones humanas expuestas al sacrificio de animales, la 
venta de carnes en expendios y la recolección de desechos, ni estudios en trabajadores de alcantarillas. La variabilidad de las prevalencias encontradas en Colombia (Don Matías Antioquia, 22.8\%, ${ }^{11}$ Cali 6.4\%, ${ }^{9}$ y departamento de Córdoba, $13.1 \%$ ) posiblemente se explican por las condiciones de saneamiento básico y el perfil ocupacional de la población estudiada.

En conclusión, el estudio permitió no sólo establecer la seroprevalencia de anticuerpos antileptospira para la población general, sino que ubicó a una población específica (Ciénaga de Oro), como zona de alta prevalencia de riesgo. Lo anterior permite focalizar las medidas preventivas y de promoción de la salud, haciendo más efectivas las intervenciones en salud pública. Por último, el trabajo aportó una información importante sobre la presencia de leptospirosis, por lo que debe ser incluida en el diagnóstico diferencial de las patologías infecciosas tropicales, que se presentan en esta región del Caribe colombiano.

\section{Referencias}

1.Zaki S, Spiegel R. Leptospirosis. En: N elson AM, H orsburgh R, Ed. Pathology of emerging infections 2.W ashington, DC:ASM Press; 1998; 73-85.

2. García E, Suárez M, García R, Pedroso S. Factores de riesgo de la leptospirosis humana en el municipio de Ciego de Avila. Rev Cub Hig Epidem 2001;39:207-213.

3. Levett P. Leptospirosis. Clin Microbiol Rev.Am soc Microb 2001;14: 296-326.

4. Acosta H, Moreno C, Viáfara D. Leptospirosis. Revisión de tema. Col Med 1994:25:36-42.

5. Angnani R, Pathak A, Mishra M. Prevalence of leptospirosis in various risk groups. Indian J Med Microb 2003;21:1-5.

6. Kaufmann A, W eyant R. Leptospiraceae. En: Balows A, Hausler W, Herrmann K, Isenberg H, Shadomy H, Ed. Manual of clinical microbiology. W ashington, DC:ASM Press; 1995:621-626.
7. A cha P, Szyfress B. Zoonosis y enfermedades transmisibles comunes a los hombres y a los animales. W ashington, DC: 0 rganización Panamericana de la Salud; 1977:57-62.

8. Mandell G , Bennett J, D alin R. Enfermedades infecciosas: principios y práctica. 4a edición. Buenos Aires: Editorial Médica Panamericana; 1997:2396-2403

9. Ferro B, Rodríguez A, Pérez M,Varona M, Gómez L. Diagnóstico de leptospirosis en la vigilancia epidemiológica en Cali. Biomedica 2003:23:26-27.

10. W inslow W, Merry D, Pirc M, D evine P. Evaluation of a commercial enzyme- linked inmunosorbent assay detection of immunoglobulin $M$ antibody in diagnosis of human leptospiral infection. J C lin Microbiol 1997;35:1938-1942.

11.0 choa J, Sánchez A, Ruiz I. Epidemiología de la leptospirosis en una zona andina de producción pecuaria. Infectio 2001;5:1-15.

12. Barguil A,Tamayo L. Prevalencia de leptospirosis porcina en el municipio de Cotorra (trabajo de grado, Facultad de Medicina Veterinaria). Montería: Universidad de Córdoba; 2000.

13. Almenteros C , A rrieta G, Máttar S. Seroprevalencia de leptospirosis porcina en el departamento de Córdoba: un riesgo para la salud pública. Rev Col Cien Pec 2004;17:141-147.

14. Bedoya Z, Mendoza S. Prevalencia de leptospirosis porcina en el municipio de Ciénaga de 0 ro, Córdoba (trabajo de grado, Facultad de Medicina Veterinaria). Montería: U niversidad de Córdoba; 2000. 15. Babilonia D, Alvarez L, N ájera S,Villera P, Máttar S. Informe preliminar de seroprevalencia de leptospirosis humana en el departamento de Córdoba. Infectio 2003;7:106.

16. Cruz R, Fernández F,A révalo H. Hiperendemicidad de leptospirosis $\mathrm{y}$ factores de riesgo asociados en localidades arroceras del departamento de San Martín - Perú. Peru Med 2002;19:10-17. 17.Adler B, Murphy A, Locarine S, Faine S. Detection of specific antileptospiral inmunoglobulins $M$ and $G$ in human serum by solidphase-enzyme linked inmunosorbent assay.J Clin Microbiol 1980; 11:452-457.

18. Meites E, Jay M, D eresinski S, Shieh W, Zaki S, Thompkins L et al. Reemerging leptospirosis in California. Emerg Infect D is 2004;10: 406-411.

19. Phraisuwan $P$, Spott E, Tharmaphornpilas P, G uharat $S$, Thongkamsamut S,A resagig $S$ et al. Leptospirosis: Skin wounds and control strategies, Thailand. Emerg Infect D is 2002;8:1435-1439. 20. Johnson M, Smith H, Joseph P, G ilman R, Bautista C, Campos K. Environmental exposure and leptospirosis, Perú. Emerg Infect D is 2004; 10:1016-1022. 